\title{
Light-induced exacerbation of retinal degeneration in a rat model of Smith-Lemli-Opitz syndrome
}

\author{
Dana K. Vaughan ${ }^{\mathrm{a}}$, Neal S. Peachey ${ }^{\mathrm{b}}$, Michael J. Richards ${ }^{\mathrm{c}}$, Blake Buchan ${ }^{\mathrm{a}}$, and Steven J. \\ Fliesler ${ }^{\mathrm{C}}{ }^{*}$ \\ aDepartment of Biology, University of Wisconsin Oshkosh, Oshkosh, WI, USA \\ ${ }^{b}$ Cleveland VA Medical Center, and Cole Eye Institute, Cleveland Clinic Foundation, Cleveland, $\mathrm{OH}$, \\ USA \\ 'Departments of Ophthalmology and Pharmacological \& Physiological Science, Saint Louis \\ University School of Medicine, St Louis, MO, USA
}

\section{Abstract}

Potentiation of retinal degeneration by intense light exposure, and its amelioration by an antioxidant, were studied in a rat model of Smith-Lemli-Opitz syndrome (SLOS), in comparison with normal (control) Sprague-Dawley rats. The SLOS model is created by treating rats with AY9944, a selective inhibitor of cholesterol synthesis at the level of $3 \beta$-hydroxysterol- $\Delta^{7}$-reductase. A subset of rats was treated with dimethylthiourea (DMTU), a synthetic antioxidant, 24 and $1 \mathrm{hr}$ prior to light exposure. Half of the animals ( \pm DMTU) were exposed to intense, constant, green light ( $24 \mathrm{hr}, 1700 \mathrm{~lx}, 490$ $580 \mathrm{~nm}$ ), while the others were maintained in darkness. Subsequently all animals were returned to dim cyclic light (20-40 lx, 12 hr light-12 hr dark) for 2 weeks, after which electroretinograms were recorded. One eye from each rat was taken for histological and quantitative morphometric analyses; sterol analysis was performed on retinas from contralateral eyes. HPLC analysis confirmed the accumulation of 7-dehydrocholesterol (7DHC) in retinas of AY9944-treated rats; cholesterol represented $>99 \%$ of the sterol in control retinas. Histology of retinas from unexposed, AY9944treated rats (6-week-old) was normal. In contrast, age-matched, light-exposed rats exhibited massive photoreceptor cell loss in both the superior and inferior hemispheres, and concomitant rod and cone dysfunction. The severity and geographic extent of the damage was far greater than that observed in normal albino rats exposed to the same conditions. DMTU pre-treatment largely prevented these degenerative changes. These findings indicate that the AY9944-induced rat SLOS model is hypersensitive to intense light-induced retinal damage, relative to normal rats. DMTU protection against light-induced damage implicates free radical-based oxidation in the retinal degeneration process. Furthermore, the use of green light (corresponding to the absorption maxima of rhodopsin) implicates rhodopsin in the initiation of the pathobiological mechanism. We propose that generation of cytotoxic oxysterols (by-products of 7DHC oxidation) is an integral part of retinal cell death in the SLOS rat model, which is exacerbated by intense light. Furthermore, the results predict lightdependent potentiation of retinal degeneration in SLOS patients, and the possible ameliorative effects of antioxidant therapy.

\section{Keywords}

AY9944; Smith-Lemli-Opitz syndrome; retina; degeneration; light damage; antioxidant; rat 


\section{Introduction}

In combination with underlying ocular disease, intense visible light exposure has long been suspected of accelerating human retinal degeneration, though the matter is still far from settled (Smith et al., 2005; Margrain et al., 2004; Tomany et al., 2004; Wang et al., 2003; Delcourt et al., 2001). Whereas chronic light exposures are difficult to quantify in human patients, experimental animal models have provided convincing evidence in support of this notion (Radu et al., 2004; Vaughan et al., 2003). Noteworthy in this regard is the recent report by Cideciyan and colleagues (2005), regarding a naturally occurring canine model of retinitis pigmentosa, in which they stated that 'modest light levels, as used in routine clinical practice, dramatically accelerated the neurodegeneration', leading those authors to call for 'a genespecific clinical trial of light reduction in human rhodopsin disease'.

Retinal light damage involves oxidative modification of endogenous retinal lipids, proteins, and nucleic acids (Wenzel et al., 2005; Glickman, 2002; Boulton et al., 2001; Organisciak and Winkler, 1994). Genetic or environmental conditions that lead to an increase in reactive oxygen species or oxidation-prone target molecules would be expected to increase both oxidative stress and cytotoxic reactions. The Smith-Lemli-Opitz syndrome (SLOS), potentially, is just such a condition (Smith et al., 1964; cf. Battaile and Steiner, 2000; Porter, 2000; Kelly, 2000; Nowaczyk and Waye, 2001; Jira et al., 2003). SLOS arises from mutations in a gene (Dhcr7) that encodes a key enzyme in the cholesterol biosynthesis pathway, namely $3 \beta$-hydroxysterol$\Delta^{7}$-reductase (DHCR7) (Waterham and Wanders, 2000; Correa-Cerro and Porter, 2005). A hallmark of SLOS is the abnormal and excessive accumulation of 7-dehydrocholesterol (7DHC), an immediate precursor of cholesterol (Tint et al., 1994). 7DHC is extremely labile to oxidation (Girotti, 2002), far more so than is cholesterol, and oxysterols are highly toxic to cells (Chang and Phelan, 2002; Panini and Sinensky, 2001; Schroepfer, 2000; Gaoua et al., 1999).

It has been proposed that the accumulation of such oxidized molecules, particularly 7DHCderived oxysterols, in the retina makes progressive retinal degeneration likely in persons with SLOS (Fliesler, 2002; Fliesler et al., 2004). This prediction of SLOS-associated retinal degeneration is consistent with a recent study that documented electroretinographic (ERG) dysfunction in a cohort of SLOS patients, particularly with regard to deficits in rod function, including delayed activation and deactivation kinetics (Elias et al., 2003). However, with the exception of a single case report on the histopathology of the retina of a 1-month-old infant with SLOS (Kretzer et al., 1981), there are no published accounts of the morphological or ultrastructural features of the retina in SLOS, particularly in older patients with more advanced disease. Additional ophthalmic features associated with SLOS, such as cataracts, optic neuropathy, and blepheroptosis, have been reported (Atchaneeyasakul et al., 1998; Cenedella, 1996; Kretzer et al., 1981).

We previously reported the biochemical, electrophysiological, and histopathological features of the retina in a rat model of SLOS, wherein the experimental drug AY9944 was used to inhibit the same enzyme that is defective in the human disease (Fliesler et al., 1999, 2004). In the initial study (Fliesler et al., 1999), AY9944 was administered to pregnant dams after the first week of gestation, and thereafter to the progeny, thereby providing a pharmacologically induced congenital rodent model of SLOS. Unexpectedly, despite marked disruption of sterol metabolism, grossly abnormal accumulation of 7DHC and profoundly reduced levels of cholesterol in the retina and other tissues, retinal structure and function appeared normal up to one post-natal month. However, in a subsequent study (Fliesler et al., 2004), we showed that when survival time was extended up to 3 months, a progressive retinal degeneration, involving both rods and cones, was observed. In the latter case, electrophysiological deficits, including 
reduced amplitudes and delayed response times, were observed, reminiscent of the types of visual defects observed in SLOS patients (Elias et al., 2003). Histological and ultrastructural examination of the retinas revealed massive accumulation of abnormal, lipophilic inclusions in the retinal pigment epithelium (RPE), photoreceptor pyknosis and dropout, and grossly shortened rod outer segments (Fliesler et al., 2004). These findings of outer retinal degeneration correlated well with the observed ERG deficits. More recently, severe hypomyelination of the optic nerve also has been observed in this rat model of SLOS (Nagel et al., 2005).

The present work was undertaken using the pharmacologically induced Sprague-Dawley rat model of SLOS to test the hypotheses that (a) bright light exposure can accelerate the SLOSassociated retinopathy; and (b) that antioxidants [e.g. dimethylthiourea (DMTU)] can exert protective effects against this light-induced retinal damage, consistent with the underlying mechanism of the pathology being a free radical-based oxidative process.

\section{Materials and methods}

\subsection{Animals}

All animal procedures were pre-approved by institutional care and use committees and conformed to the ARVO Statement on the Care and Use of Animals in Ophthalmic and Vision Research and with the NIH Guide for the Care and Use of Laboratory Animals. The pharmacologically induced rat model of SLOS was produced as previously described (Fliesler et al., 1999, 2004). In brief, pregnant female Sprague-Dawley rats (Harlan; 6 days spermpositive) were administered AY9944 (obtained by custom synthesis) by feeding $40 \mathrm{~g} /$ day of normal powdered rodent chow laced with AY9944 ( $1 \mathrm{mg} / 100 \mathrm{~g}$ chow), starting on gestational day 7 and continuing throughout the post-partum lactation period to weaning. Pups were injected with AY9944 (30 mg/kg, in PBS) three times per week on alternating days, starting at post-natal day 1 (P1) and continuing throughout life, and were fed cholesterol-free chow (Purina Test Mills Inc.) upon weaning, ad lib. Control rats received no AY9944 and ate normal chow. All rats were maintained in dim cyclic light (20-40 lx, $12 \mathrm{hr}$ light-12 hr dark) at 22$25^{\circ} \mathrm{C}$ and were provided water ad lib.

\subsection{Light damage and DMTU treatment}

Intense light exposure (retinal light damage paradigm) was performed essentially as described in detail previously (Organisciak et al., 1996, 1999; Vaughan et al., 2003). After 20-24 hr of dark adaptation, control and AY9944-treated animals were light-exposed, either at P28 or P37 (when AY9944-treated animals show little sign of retinal degeneration; Fliesler et al., 1999). Intense light administration constituted a single, $24 \mathrm{hr}, 360^{\circ}$, constant exposure to green light (1700 lx, 490-580 nm) in a temperature-controlled chamber (kindly provided by D.T. Organisciak, Wright State University School of Medicine, Dayton, OH). Unexposed rats remained in darkness for the same $24 \mathrm{hr}$ period. A subset of AY9944-treated and control rats was given subdermal aqueous injections of the synthetic antioxidant dimethylthiourea (DMTU, Sigma-Aldrich; $500 \mathrm{mg} / \mathrm{kg}$ ) at $24 \mathrm{hr}$ prior, and again at $1 \mathrm{hr}$ prior, to the $24 \mathrm{hr}$ of intense light exposure or continued darkness (Lam et al., 1990; Organisciak et al., 1999). All rats were allowed 2 weeks recovery in dim cyclic light after exposure prior to ERG recording and sacrifice, to permit clearance of dead photoreceptors (if any) from the outer nuclear layer (ONL).

Treatment groups thus employed were: (1) controls unexposed to intense light, with and without DMTU (CUD and CU, respectively); (2) controls exposed to intense light, with and without DMTU (CED and CE, respectively); (3) AY9944-treated animals unexposed to intense light, with and without DMTU (AUD and AU, respectively); and (4) AY9944-treated animals 
exposed to intense light, with and without DMTU (AED and AE, respectively). Each group had an $N$ of 2-6 rats.

\subsection{Electrophysiology}

The methods employed for obtaining rod ERGs and their subsequent analysis were modified from those described previously (Fliesler et al., 2004), as follows. After overnight dark adaptation, rats were anesthetized by intramuscular injection of ketamine $\mathrm{HCl}(75 \mathrm{mg} / \mathrm{kg})$ and xylazine $\mathrm{HCl}(5 \mathrm{mg} / \mathrm{kg})$. Eyedrops were used to dilate the pupil of the test eye (1\% tropicamide, $2.5 \%$ phenylephrine $\mathrm{HCl}, 1 \%$ cyclopentolate) and to anesthetize the corneal surface ( $1 \%$ proparacaine $\mathrm{HCl}$ ). Rats were placed on a heating pad throughout the recording session. ERGs were recorded using a stainless steel electrode contacting the corneal surface through a thin layer of $1 \%$ methylcellulose. Needle electrodes placed in the cheek and tail served as reference and ground leads, respectively. The signal was differentially amplified (bandpass filter of 1$1000 \mathrm{~Hz}$ ), digitized, and stored using a Nicolet (Madison, WI) Compact Four signal averaging system. From each rat, a dark-adapted response series was obtained using strobe flash stimuli that ranged in intensity from -3.2 and $0.5 \log \mathrm{cd} \mathrm{sec} / \mathrm{m}^{2}$. Strobe flash stimuli were presented in order of increasing intensity within a Nicolet ganzfeld bowl, and responses to two successive flashes were averaged. A $30 \mathrm{sec}$ interflash interval was used for low stimulus intensities while a 1 min interval was used for the higher stimulus flashes. The amplitude of the a-wave was measured from the pre-stimulus baseline to the trough of the a-wave. The amplitude of the bwave was measured from the trough of the a-wave or, if no a-wave was present from the baseline to the peak. Implicit time was measured from the time of flash presentation to the awave trough or the b-wave peak. At the completion of the recording session, the deeply anesthetized rats were sacrificed by decapitation and tissues were harvested for biochemical and histological analyses.

\subsection{Biochemistry}

Methods utilized for harvesting neural retinas and saponification, extraction, and chromatographic analysis of their constituent sterols were as described in detail elsewhere (Fliesler et al., 1999, 2000, 2004). All procedures were performed under dim room illumination to minimize light-induced isomerization and degradation of lipids. In brief, one neural retina from each animal was saponified with methanolic $\mathrm{KOH}$ under argon atmosphere, and the nonsaponifiable lipids (the sterol-containing fraction) were extracted with petroleum ether. The non-saponifiable extracts were then analysed by reverse-phase HPLC, with detection by flowthrough spectrophotometry (detection at $205 \mathrm{~nm}$ ). To correct for losses incurred during initial tissue processing, specimens were supplemented with an internal standard of $\left[{ }^{3} \mathrm{H}\right]$ cholesterol (American Radiolabeled Chemicals Inc.) immediately prior to saponification. Identification and quantification of sterols were performed by comparison with authentic sterol standards, particularly cholesterol and 7DHC (Sigma-Aldrich; recrystallized from methanol-water twice before use).

\subsection{Histological and quantitative morphometric analysis}

The methods employed for fixation, embedment, sectioning, and histological analysis of eyes were as previously described (Vaughan et al., 2003; Fliesler et al., 2004). In brief, one eye of each animal, fixed with buffered mixed aldehydes, was dissected into hemispheres such that the vertical meridian from superior to inferior pole became the sectioned face. The hemispheres were post-fixed in osmium, serially dehydrated, and embedded in Spurr's resin by standard methods. The block angle of embedded eyes was adjusted to achieve optimal alignment of photoreceptor outer segments. Three semithin sections $(0.75 \mu \mathrm{m}$ thickness $)$ spanning the entire hemisphere were mounted on glass microscope slides and stained with $1 \%$ toluidine blue for standard light microscopy. Representative images at defined loci along the vertical meridian 
were photographed using a digital imaging system (Nikon DXM1200) attached to an Olympus BH-2 photomicroscope, at various magnifications, and stored on a computer.

Quantitative morphometric analysis was performed as described in detail previously (Fliesler et al., 2004). In brief, to quantify photoreceptor survival, measurements of ONL thickness were taken at $0.5 \mathrm{~mm}$ intervals along the vertical meridian, from the optic nerve head and extending to the inferior and superior margins. Five ONL thickness measurements were made at each location per tissue section, using video imaging and photomicroscopy equipment, and the data were imported into a computer spreadsheet (Microsoft Excel ${ }^{\circledR}$ ). Data from each locus along the vertical meridian were averaged using three serial sections per eye in each treatment group and expressed as mean ONL thickness $( \pm \mathrm{SD})$ as a function of eccentricity from the optic nerve head. Mean ONL thickness across the entire vertical meridian was also calculated to provide a summary measure of photoreceptor damage per treatment group. These data were compared by small-sample statistical analysis (Student's $t$-test, two-tailed, assuming equal variances). Significance was determined at $p<0.001$ unless otherwise indicated.

\section{Results}

\subsection{Electrophysiology}

Fig. 1A presents representative dark-adapted ERGs depicting responses to a series of five increasing flash intensities for each experimental condition employed. As noted previously for animals reared under cyclic lighting conditions (Fliesler et al., 2004), responses obtained from AY9944-treated rats not exposed to intense light (AU) were reduced in amplitude in comparison to control rats (CU). In fact, the extent of the reduction in response amplitudes was comparable to that observed for control rats exposed to intense green light (CE). Notably, however, this reduction in rod ERG response amplitudes preceded the appearance of frank histological abnormalities (see below). Furthermore, when AY9944-treated rats were exposed to intense green light (AE), the effect was even more dramatic; rod-dependent responses were profoundly diminished, relative both to non-light-exposed rats $(\mathrm{CU}, \mathrm{AU})$ and to light-exposed controls (CE).

These qualitative data were extended by quantitative analysis. Fig. 1B-D presents average $( \pm 1$ $\mathrm{SD})$ intensity-response data for the main ERG components. In rats that were not exposed to high intensity light, the ERGs of AY9944-treated rats were smaller than those of control rats. The overall reduction was relatively modest. ERGs were smaller in all rats exposed to high intensity light. For example, across all flash intensities, the amplitude of the a-wave in CE rats was $84.9 \pm 8.4 \%$ of that in CU rats (Fig. 1B). The greatest reduction was noted in AY9944treated animals. For example, the amplitude of the a-wave in AE rats was only $5.8 \pm 4.7 \%$ of that in AU rats. Similar effects were observed for the b-wave (Fig. 1D). In control rats, across the flash intensity series, the amplitude of the b-wave in CE rats was $85.1 \pm 5.0 \%$ of that in $\mathrm{CU}$ rats. In AY9944-treated rats, the amplitude of the b-wave in AE rats was $16.6 \pm 4.3 \%$ of that in AU rats. Notably, AY9944-treated rats had delayed b-wave implicit times (Fig. 1C), in good agreement with our prior findings (Fliesler et al., 2004), and these delays were exacerbated by intense light exposure.

\subsection{Biochemistry}

As shown in Fig. 2, the central biochemical hallmark of SLOS was achieved under the conditions of AY9944 treatment: in the treated groups (AU, AE), 7DHC accumulated in retinas to a level essentially comparable to that of cholesterol, whereas retinas of rats in the control groups (CU, CE) contained essentially no 7DHC. Comparing the two control groups, plus and minus exposure to intense light (CU vs. CE), there was a significant (ca. 20\%) reduction in the amount of retinal cholesterol (essentially equivalent to that of total sterols), consistent with the 
mean reduction in tissue mass due to the light-induced retinal degeneration (see below). In comparing the $\mathrm{CU}$ vs. $\mathrm{AU}$ groups, as well as the $\mathrm{CE}$ vs. AE groups, the total sterol content of retinas was reduced by ca. $25 \%$ in the AY9944-treated rats; this is largely due to the diminished growth rate and body mass of the treated animals, relative to controls, which is mirrored by parallel differences in eye size, consistent with prior findings (Fliesler et al., 1999). In the AY9944-treated groups, the gain in 7DHC content of the retinas, relative to controls, is balanced by the reduction in cholesterol content, consistent with a one-for-one molar replacement of cholesterol by 7DHC.

\subsection{Histology and quantitative morphometry}

Qualitative inspection by light microscopy revealed no perceptible differences between CU and AU retinas, particularly with regard to ONL thickness, ONL pyknosis, or rod outer segment (ROS) layer thickness or general appearance (data not shown). Fig. 3 shows low magnificent images (taken with a $2.5 \times$ objective lens) of toluidine blue-stained retinal sections along the vertical meridian, taken from eyes of rats in the CE (Fig. 3A) and AE (Fig. 3B) groups. To the right of each of these low magnification images is a four-panel series of corresponding higher magnification images (taken with a 20x objective lens), taken 1 and $3 \mathrm{~mm}$ from the optic nerve head in both the superior (upper panels) and inferior (lower panels) hemispheres. Even at low magnification, one can see the deep purple staining corresponding to photoreceptor nuclei in the ONL; with exposure to intense light, an obviously more pale zone is observed in the ONL where photoreceptor loss has occurred. In the latter zones, remnant photoreceptors consisting mostly of severely pyknotic cells completely lacking ROS are observed. At the time of sacrifice, these animals were ca. 6-week-old; hence, these findings are comparable to those previously observed in 4-week-old AY9944-treated rats (Fliesler et al., 1999), but are unlike those observed in 10-week-old AY9944-treated rats (Fliesler et al., 2004), where retinal degeneration was observed. As expected, photoreceptor loss from CE rat retinas was modest, with severe cellular damage and loss being relatively restricted geographically to the superior central region, in complete agreement with the prior findings of several other labs (see Wenzel et al., 2005; Glickman, 2002;Boulton et al., 2001;Organisciak and Winkler, 1994). Retinal histology in the inferior central zone and in the peripheral retina of both hemispheres appeared relatively normal. In striking contrast, AE rat retinas exhibited massive photoreceptor loss from both hemispheres, with a disproportionately greater loss from the superior hemisphere, extending well beyond the central retinal region into the mid-periphery (Fig. 3B). Thus, AY9944 administration greatly potentiated photoreceptor loss due to intense light exposure, both in severity and in geographic extent, and such light exposure dramatically hastened the kinetics of the natural time course of retinal degeneration observed in this SLOS rat model.

Morphometry confirmed and extended these qualitative histological observations and provided clear location-specific information regarding the extent of light-induced retinal damage (see plot of ONL thickness as a function of distance from the optic nerve head, Fig. 4). The severity and geographic distribution of light damage was far greater in AY9944-treated rats compared to controls: virtually complete photoreceptor loss was observed across the entire central to midperipheral superior retina, as well as in the inferior central retina (with more moderate photoreceptor loss in the mid-peripheral inferior hemisphere), in the AE group. By comparison, the CE group exhibited only partial devastation of photoreceptors in the superior central zone, with only moderate to minimal photoreceptor loss extending to the mid-periphery, and much less reduction in ONL thickness in the inferior retina, compared to the superior hemisphere. In addition, systemic pre-treatment of AY9944-treated rats with DMTU markedly spared the degeneration and loss of photoreceptors (cf. AE vs. AED, Fig. 4). Across the vertical meridian, in the absence of intense light exposure, DMTU pre-treatment had no significant effect $(p>0.05)$ on mean ONL thickness in non-light-exposed control (CU) or AY9944-treated (AU) rats. However, in AY9944-treated animals, DMTU administration prior to intense light 
exposure was significantly protective, resulting in only a $23 \%$ reduction of mean ONL thickness (AED; $p<0.01$ vs. AU), compared to a $73 \%$ reduction when DMTU was not used (AE; $p<0.001$ vs. AU). The sparing effect of DMTU observed in these experiments was even greater than that previously reported for normal albino rats treated similarly (see Organisciak et al., 1999).

\section{Discussion}

Herein, we have demonstrated that AY9944 treatment of rats renders their retinas extraordinarily susceptible to the damaging effects of intense light exposure. In normal albino rats, under the conditions employed in this study (1700 lx, 490-580 nm light, $24 \mathrm{hr}$ exposure following 24-hr dark adaptation), photoreceptor cell death and loss are largely localized to the superior central retina (Wenzel et al., 2005; Glickman, 2002; Boulton et al., 2001; Organisciak and Winkler, 1994). However, in AY9944-treated albino rats, the zone of photoreceptor death and loss extends to the mid-peripheral retina in both the superior and inferior hemispheres, with essentially total loss of photoreceptors in the central zone. In addition, the ERG data suggest that, by the sixth post-natal week, photoreceptors and other retinal neurons in the SLOS rat model are functionally compromised even before any obvious histological abnormalities are evident, since rod responses in the AU group were comparable to those in the CE group. Hence, these observations provide an additional time point within the course of progressive retinal degeneration in the SLOS rat model, intermediate between the 1-month time point, where no appreciable structural or functional damage is evident (Fliesler et al., 1999), and the 3-month time point, where extensive retinal degeneration and dysfunction are observed (Fliesler et al., 2004).

These results beg at least two central questions: (1) why does retinal degeneration occur at all in the SLOS rat model, and (2) why does exposure to intense green light cause far more extensive retinal damage than observed in normal albino rats? In a previous study (Richards et al., 2000), ROS and other retinal membranes of AY9944-treated rats exhibited profoundly altered fatty acid composition, relative to age-matched controls fed the same diet (minus AY9944). Remarkably, ROS docosahexaenoic acid (DHA; C22:603) levels were reduced by nearly $21 \%$ in AY9944-treated rats, relative to controls, a feat which had only been achieved previously by feeding animals an $\omega 3$-deficient diet (Bush et al., 1991; Remé et al., 1994; Koutz et al., 1995; Organisciak et al., 1996). In those prior studies, it was shown that lowering the levels of retinal DHA results in a reduced susceptibility to retinal light damage, reasonably predicting that AY9944-treated animals should also exhibit reduced susceptibility to intense light-induced retinal degeneration. However, as clearly shown in the present study, AY9944treated rats are hypersensitive to retinal light damage. This suggests that something other than polyunsaturated fatty acid status is more central to the mechanism of retinal degeneration in the SLOS rat model. We propose that the key lies in the nature of the sterol that accumulates in the retina and other tissues in the SLOS rat model.

As demonstrated heretofore (Fliesler et al., 1999, 2004) and in the present study, AY9944treated rats accumulate 7DHC in their retinas, whereas normal rat retinas contain essentially no steady-state levels of this cholesterol precursor. 7DHC is well-known to be extremely prone to autooxidation (far more so than is cholesterol), particularly when exposed to light, generating oxysterol products that are extremely cytotoxic (Girotti, 2002). The retina (particularly the photoreceptor cells) is exposed to a high flux of oxygen (Cringle et al., 1991, 2002; Yu and Cringle, 2005). Hence, it is reasonable to suspect that the in situ formation of 7DHC in a highly oxidizing environment would result in the progressive conversion of at least some of the 7DHC to oxysterols, which would then compromise cellular functions and viability, ultimately resulting in the observed retinal degeneration. It is known that the levels of oxysterol required to induce various pathological sequellae are extremely small, compared to the steady-state 
levels of the parent sterol (Schroepfer, 2000; Panini and Sinensky, 2001; Chang and Phelan, 2002). Since such oxysterol formation is potentiated by light exposure, one might think that this would explain the observed exacerbation of retinal damage in the SLOS rat model by intense light. However, such light-augmented oxidation of 7DHC is restricted primarily to UVA wavelengths, as opposed to the green visible light $(490-580 \mathrm{~nm})$ used in the present study. Since rhodopsin is, by far, the dominant green-absorbing molecule in the albino rat retina and the action spectrum of retinal light damage corresponds to the absorption spectrum of rhodopsin (see Organisciak and Winkler, 1994, and references cited therein), the light-induced exacerbation of the SLOS rat retinal degeneration likely is rhodopsin-mediated. Hence, in agreement with current models implicating lipid peroxidation in the mechanism of retinal light damage (Wenzel et al., 2005; Glickman, 2002; Boulton et al., 2001; Organisciak and Winkler, 1994), we speculate that light absorption by rhodopsin triggers the formation of pro-oxidants, which cause oxidation of 7DHC to cytotoxic oxysterols. Since 7DHC is appreciably absent from normal retinas, this explains the hypersensitivity to retinal light damage observed in the SLOS rat model compared to normal albino rats.

Further support for this speculation comes from two independent lines of evidence. First, from the present study, administration of the synthetic antioxidant DMTU prior to intense light exposure provided almost complete protection from light-induced photoreceptor loss. DMTU is a hydroxyl radical scavenger (Fox et al., 1983; Harada et al., 1983; Parker et al., 1985); hence, as is suspected of retinal light damage in normal albino rats, the light-exacerbated retinal degeneration observed in the SLOS rat model likely involves free radical-induced oxidation. Second, we have demonstrated (Richards et al., 2005) a correlation between the accumulation of lipid hydroperoxides and the extent of retinal degeneration in the SLOS rat model, under both normal (low-level) lighting conditions and under conditions of intense green light exposure. In fact, the levels of lipid hydroperoxides in the retinas of light-damaged albino rats were comparable to those of non-light-damaged, AY9944-treated albino rats (both of which were ca. two-fold higher than lipid hydroperoxide levels found in control, non-light-damaged albino rat retinas), and intense light exposure tripled these levels in the SLOS rat model. In preliminary studies (S.J. Fliesler and M.J. Richards, unpublished), we have found that exceedingly small amounts of 7DHC-derived oxysterols (0.1-5 nmoles) react robustly in the lipid hydroperoxide assay (Cayman LPO Detection Kit), far more so, mole for mole, than do peroxidized fatty acids, whereas 7DHC, cholesterol, and common cholesterol-derived oxysterols (e.g. 25-hydroxycholesterol, 7-ketocholesterol, 7-hydroxycholesterol) do not. This suggests that very little conversion of 7DHC to cytotoxic oxysterols would be required to induce the observed retinal degeneration in the SLOS rat model. Consistent with this, neither the absolute level of 7DHC nor the 7DHC/Chol mole ratio is appreciably diminished in retinas of light-damaged vs. non-light-exposed, AY9944-treat rats (see Fig. 2), yet there is a remarkable difference in the extent of retinal damage under the two conditions. While we did not measure the levels of 7DHC-derived oxysterols directly in these studies, such is the focus of our ongoing research efforts.

To the extent that the AY9944-induced rat model faithfully mimics certain essential features of SLOS, our findings may have significance to this devastating, hereditary human disease. For one, our results predict that the retinas of SLOS patients may be hypersensitive to the damaging effects of intense light exposure, and should be protected from such. It is documented that SLOS patients are extremely photosensitive, dermatologically (Charman et al., 1998; Anstey et al., 1999). Secondly, we propose that antioxidant administration, in addition to the current therapeutic strategy of cholesterol supplementation, may be therapeutically beneficial in human SLOS patients. Although acute DMTU administration is protective against retinal light damage in animal model studies, it cannot be used in humans, as it is toxic (Saillenfait et al., 1991). Instead, alternative, more biologically compatible antioxidants should be considered 
(e.g. vitamins $\mathrm{E}$ and $\mathrm{C}, \alpha$-lipoic acid, etc.). Toward this end, studies are currently underway in our laboratory to evaluate the protective effects of various antioxidants in the SLOS rat model.

\section{Acknowledgments}

This work was supported, in part, by: the UW Oshkosh Faculty Development Program (DKV); the UW Oshkosh Undergraduate Collaborative Grant Program (BB); the Medical Research Service, Department of Veterans Affairs (NSP); U.S.P.H.S. grant EY07361 (SJF, MJR); the March of Dimes (SJF, MJR); and by an unrestricted departmental grant from Research to Prevent Blindness (SJF, MJR). Authorship of this manuscript conforms to the ICMJE's Uniform Requirements for Manuscripts Submitted to Biomedical Journals. The authors gratefully acknowledge Dr Daniel T. Organisciak for graciously providing the light damage chamber used in this study.

\section{References}

Anstey AV, Ryan A, Rhodes LE, Charman CR, Arlett CF, Tyrrell RM, Taylor CR, Pearse AD. Characterization of photosensitivity in the Smith-Lemli-Opitz syndrome: a new congenital photosensitivity syndrome. Br J Dermatol 1999;141:406-414. [PubMed: 10583043]

Atchaneeyasakul LO, Linck LM, Connor WE, Weleber RG, Steiner RD. Eye findings in eight children and a spontaneously aborted fetus with RSH/Smith-Lemli-Opitz syndrome. Am J Med Genet 1998;80:501-505. [PubMed: 9880216]

Battaile KP, Steiner RD. Smith-Lemli-Opitz syndrome: the first malformation syndrome associated with defective cholesterol synthesis. Mol Genet Metab 2000;71:154-162. [PubMed: 11001806]

Boulton M, Rozanowska M, Rozanowski B. Retinal photodamage. J Photochem Photobiol B 2001;64:144-161. [PubMed: 11744401]

Bush RA, Remé CE, Malnoe A. Light damage in the rat retina: the effect of dietary deprivation of N-3 fatty acids on acute structural alterations. Exp Eye Res 1991;53:741-752. [PubMed: 1838336]

Cenedella RJ. Cholesterol and cataracts. Surv Ophthalmol 1996;40:320-337. [PubMed: 8658343]

Chang, JY.; Phelan, KD. Effects of oxysterols on cells of the nervous system. In: Fliesler, SJ., editor. Sterols and Oxysterols: Chemistry, Biology, and Pathobiology. Research Signpost; Trivandrum, India: 2002. p. 175-200.

Charman CR, Ryan A, Tyrrell RM, Pearse AD, Arlett CF, Kurwa HA, Shortland G, Anstey A. Photosensitivity associated with the Smith-Lemli-Opitz syndrome. Br J Dermatol 1998;138:885-888. [PubMed: 9666840]

Cideciyan AV, Jacobson SG, Aleman TS, et al. In vivo dynamics of retinal injury and repair in the rhodopsin mutant dog model of human retinitis pigmentosa. Proc Natl Acad Sci USA 2005;102:52335238. [PubMed: 15784735]

Correa-Cerro LS, Porter FD. 3 $\beta$-hydroxysterol $\Delta 7$-reductase and the Smith-Lemli-Opitz syndrome. Mol Genet Metab 2005;84:112-126. [PubMed: 15670717]

Cringle SJ, Yu DY, Alder VA. Intraretinal oxygen tension in the rat eye. Graefes Arch Clin Exp Ophthalmol 1991;229:574-577. [PubMed: 1765301]

Cringle SJ, Yu DY, Yu PK, Su EN. Intraretinal oxygen consumption in the rat in vivo. Invest Ophthalmol Vis Sci 2002;43:1922-1927. [PubMed: 12037000]

Delcourt C, Carriere I, Ponton-Sanchez A, Fourrey S, Lacroux A, Papoz L. Light exposure and the risk of age-related macular degeneration: the Pathologies Oculaires Liees a l'Age (POLA) study. Arch Ophthalmol 2001;119:1463-1468. [PubMed: 11594945]

Elias ER, Hansen RM, Irons M, Quinn NB, Fulton AB. Rod photoreceptor responses in children with Smith-Lemli-Opitz syndrome. Arch Ophthalmol 2003;121:1738-1743. [PubMed: 14662594]

Fliesler, SJ. Effects of cholesterol biosynthesis inhibitors on retinal development, structure, and function. In: Fliesler, SJ., editor. Sterols and Oxysterols: Chemistry, Biology, and Pathobiology. Research Signpost; Trivandrum, India: 2002. p. 77-109.

Fliesler SJ, Richards MJ, Miller C-Y, Peachey NS. Marked alteration of sterol metabolism and composition without compromising retinal development or function. Invest Ophthalmol Vis Sci 1999;40:1792-1801. [PubMed: 10393050]

Exp Eye Res. Author manuscript; available in PMC 2010 March 24. 
Fliesler SJ, Richards MJ, Miller C-Y, Peachey NS, Cenedella RJ. Retinal structure and function in an animal model that replicates the biochemical hallmarks of desmosterolosis. Neurochem Res 2000;25:685-694. [PubMed: 10905631]

Fliesler SJ, Peachey NS, Richards MJ, Nagel BA, Vaughan DK. Retinal degeneration in a rodent model of Smith-Lemli-Opitz syndrome: electrophysiologic, biochemical, and morphologic features. Arch Ophthalmol 2004;122:1190-1200. [PubMed: 15302661]

Fox RB, Harada RN, Tate RM, Repine JE. Prevention of thiourea-induced pulmonary edema by hydroxylradical scavengers. J Appl Physiol 1983;55:1456-1459. [PubMed: 6417079]

Gaoua W, Chevy F, Roux C, Wolf C. Oxidized derivatives of 7-dehydrocholesterol induce growth retardation in cultured rat embryos: a model for antenatal growth retardation in the Smith-LemliOpitz syndrome. J Lipid Res 1999;40:456-463. [PubMed: 10064734]

Girotti, AW. Cholesterol-derived hydroperoxides: generation and reactivity in biological systems. In: Fliesler, SJ., editor. Sterols and Oxysterols: Chemistry, Biology, and Pathobiology. Research Signpost; Trivandrum, India: 2002. p. 121-139.

Glickman RD. Phototoxicity to the retina: mechanisms of damage. Int J Toxicol 2002;21:473-490. [PubMed: 12537644]

Harada RN, Vatter AE, Repine JE. Oxygen radical scavengers protect alveolar macrophages from hyperoxic injury in vitro. Am Rev Respir Dis 1983;128:761-762. [PubMed: 6414352]

Jira PE, Waterham HR, Wanders RJ, Smeitink JA, Sengers RC, Wevers RA. Smith-Lemli-Opitz syndrome and the DHCR7 gene. Ann Hum Genet 2003;67:269-280. [PubMed: 12914579]

Kelley RI. Inborn errors of cholesterol biosynthesis. Adv Pediatr 2000;47:1-53. [PubMed: 10959439]

Koutz CA, Wiegand RD, Rapp LM, Anderson RE. Effect of dietary fat on the response of the rat retina to chronic and acute light stress. Exp Eye Res 1995;60:307-316. [PubMed: 7789410]

Kretzer FL, Hittner HM, Mehta RS. Ocular manifestations of the Smith-Lemli-Opitz syndrome. Arch Ophthalmol 1981;99:2000-2006. [PubMed: 7295150]

Lam S, Tso MO, Gurne DH. Amelioration of retinal photic injury in albino rats by dimethylthiourea. Arch Ophthalmol 1990;108:1751-1757. [PubMed: 2124103]

Margrain TH, Boulton M, Marshall J, Sliney DH. Do blue light filters confer protection against agerelated macular degeneration? Prog Retin Eye Res 2004;23:523-531. [PubMed: 15302349]

Nagel, BA.; Richards, MJ.; Fliesler, SJ. Optic nerve hypomyelination in a rat model of Smith-Leml-Opitz Syndrome. Abstract \#3166, Annual Meeting Abstract Search and Program Planner [on CD-ROM], Association for Research in Vision and Ophthalmology; 2005.

Nowaczyk MJ, Waye JS. The Smith-Lemli-Opitz syndrome: a novel metabolic way of understanding developmental biology, embryogenesis, and dysmorphology. Clin Genet 2001;59:375-386. [PubMed: 11453964]

Organisciak DT, Winkler BS. Retinal light damage: practical and theoretical considerations. Prog Ret Eye Res 1994;13:1-29.

Organisciak DT, Darrow RA, Jiang Y-L, Blanks JC. Retinal light damage in rats with altered levels of rod outer segment docosahexaenoate. Invest Ophthalmol Vis Sci 1996;37:2243-2257. [PubMed: 8843911]

Organisciak DT, Darrow RA, Barsalou L, Darrow RM, Lininger LA. Light-induced damage in the retina: differential effects of dimethylthiourea on photoreceptor survival, apoptosis and DNA oxidation. Photochem Photobiol 1999;70:261-268. [PubMed: 10461466]

Panini SR, Sinensky MS. Mechanisms of oxysterol-induced apoptosis. Curr Opin Lipidol 2001;12:529533. [PubMed: 11561172]

Parker NB, Berger EM, Curtis WE, Muldrow ME, Linas SL, Repine JE. Hydrogen peroxide causes dimethylthiourea consumption while hydroxyl radical causes dimethyl sulfoxide consumption in vitro. J Free Radic Biol Med 1985;1:415-419. [PubMed: 3018065]

Porter FD. RSH/Smith-Lemli-Opitz syndrome: a multiple congenital anomaly/mental retardation syndrome due to an inborn error of cholesterol biosynthesis. Mol Genet Metab 2000;71:163-174. [PubMed: 11001807]

Radu RA, Mata NL, Bagla A, Travis GH. Light exposure stimulates formation of A2E oxiranes in a mouse model of Stargardt's macular degeneration. Proc Natl Acad Sci USA 2004;101:5928-5933. [PubMed: 15067110] 
Remé CE, Malnoe A, Jung HH, Wei Q, Munz K. Effect of dietary fish oil on acute light-induced photoreceptor damage in the rat retina. Invest Ophthalmol Vis Sci 1994;35:78-90. [PubMed: 8300366]

Richards MJ, Fliesler SJ, McClellan M, Maude MB, Anderson RE. 'Sterol synergism' in the retina: perturbation of fatty acid composition of retinal membranes as a consequence of altered sterol composition. Invest Ophthalmol Vis Sci (ARVO Abstr) 2000;41:S880.

Richards MJ, Nagel BA, Fliesler SJ. Lipid hydroperoxide formation in the retina: correlation with retinal degeneration and light damage in a rat model of Smith-Lemli-Opitz syndrome. Exp Eye Res. 2005; (this issue)10.1016/j.exer.2005.08.016

Saillenfait AM, Sabate JP, Langonne I, de Ceaurriz J. Difference in the developmental toxicity of ethylenethiourea and three $\mathrm{N}, \mathrm{N}^{\prime}$-substituted thiourea derivatives in rats. Fundam Appl Toxicol 1991;17:399-408. [PubMed: 1765227]

Schroepfer GJ Jr. Oxysterols: modulators of cholesterol metabolism and other processes. Physiol Rev 2000;80:361-554. [PubMed: 10617772]

Smith BT, Belani S, Ho AC. Light energy, cataract surgery, and progression of age-related macular degeneration. Curr Opin Ophthalmol 2005;16:166-169. [PubMed: 15870573]

Smith JD, Lemli L, Opitz JM. A newly recognized syndrome of multiple congenital anomalies. J Pediatr 1964;64:210-217. [PubMed: 14119520]

Tint GS, Irons M, Elias ER, et al. Defective cholesterol biosynthesis associated with the Smith-LemliOpitz syndrome. N Engl J Med 1994;330:107-113. [PubMed: 8259166]

Tomany SC, Cruickshanks KJ, Klein R, Klein BE, Knudtson MD. Sunlight and the 10-year incidence of age-related maculopathy: the Beaver Dam Eye Study. Arch Ophthalmol 2004;122:750-757. [PubMed: 15136324]

Vaughan DK, Coulibaly SF, Darrow RM, Organisciak DT. A morphometric study of light-induced damage in transgenic rat models of retinitis pigmentosa. Invest Ophthalmol Vis Sci 2003;44:848855. [PubMed: 12556421]

Wang JJ, Jakobsen K, Smith W, Mitchell P. Five-year incidence of age-related maculopathy in relation to iris, skin or hair colour, and skin sun sensitivity: the Blue Mountains Eye Study. Clin Experiment Ophthalmol 2003;31:317-321. [PubMed: 12880456]

Waterham HR, Wanders RJ. Biochemical and genetic aspects of 7-dehydrocholesterol reductase and Smith-Lemli-Opitz syndrome. Biochim Biophys Acta 2000;1529:340-356. [PubMed: 11111101]

Wenzel A, Grimm C, Samardzija M, Reme CE. Molecular mechanisms of light-induced photoreceptor apoptosis and neuroprotection for retinal degeneration. Prog Retin Eye Res 2005;24:275-306. [PubMed: 15610977]

Yu DY, Cringle SJ. Retinal degeneration and local oxygen metabolism. Exp Eye Res 2005;80:745-751. [PubMed: 15939030]

Exp Eye Res. Author manuscript; available in PMC 2010 March 24. 


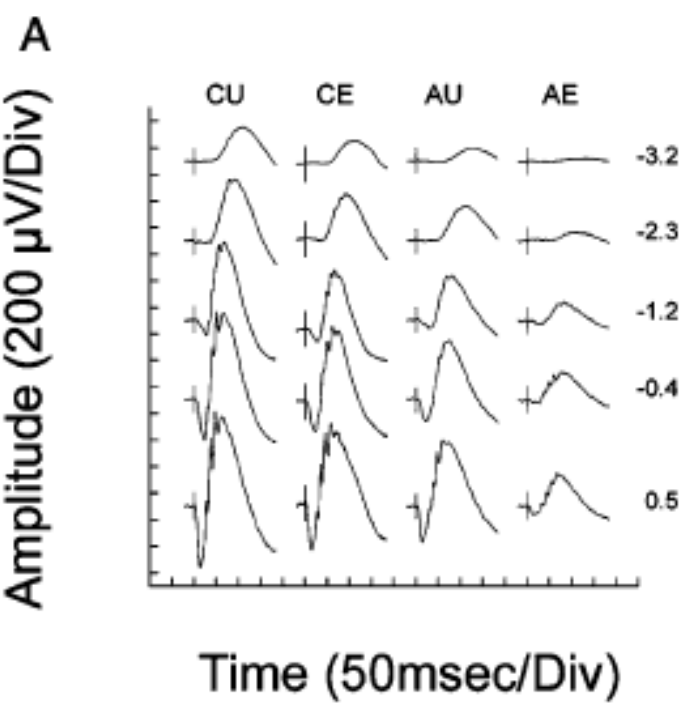

C

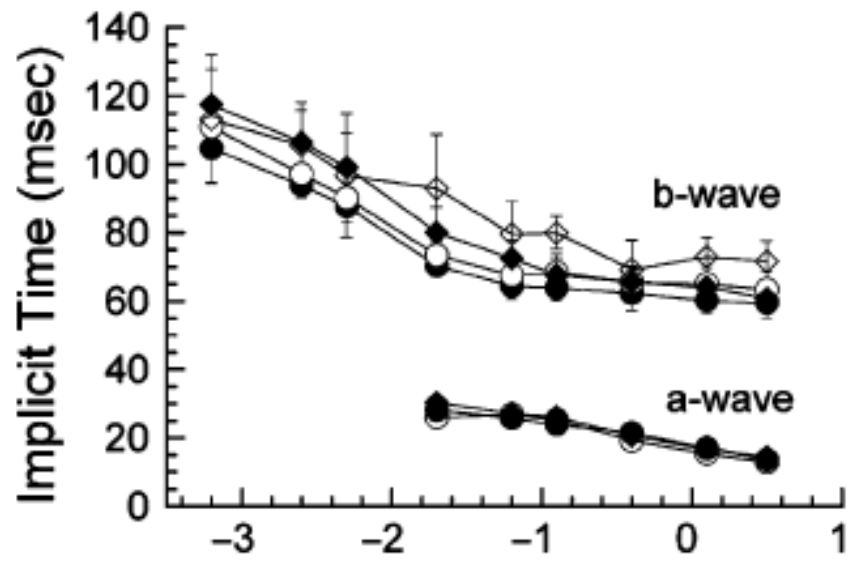

Flash Intensity (log cd sec/m $\left.\mathrm{m}^{2}\right)$
B

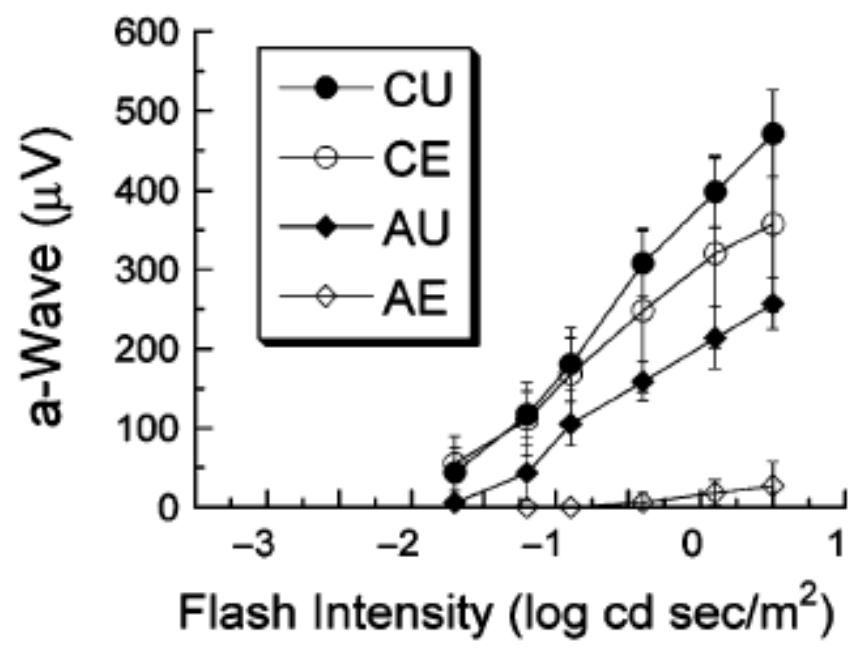

D

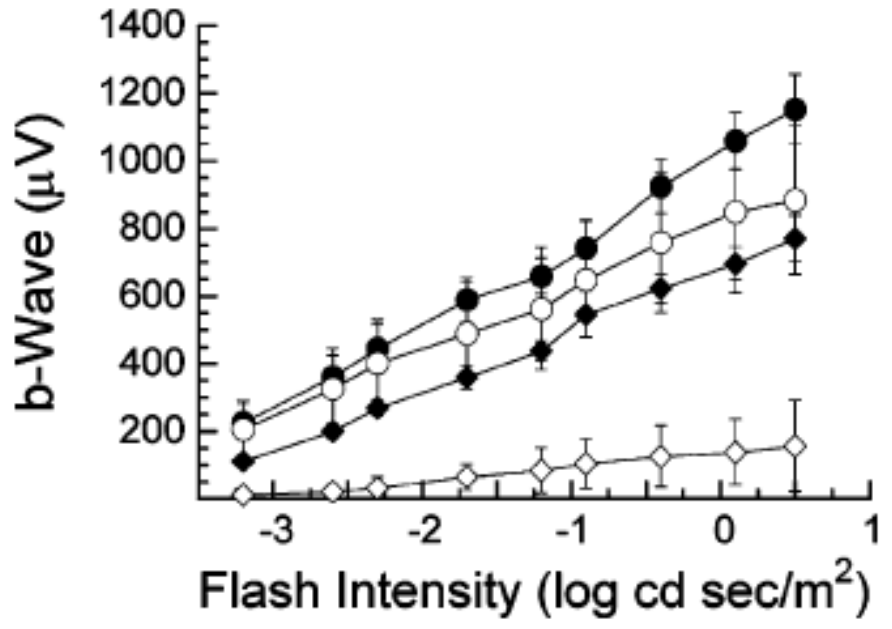

Fig. 1.

Electrophysiological recordings (ERGs). (A) Representative ERGs obtained from a rat in each experimental group. (B) Intensity-response functions for a-wave amplitudes. (C) a- and bwave implicit times. (D) b-wave amplitudes. Data points denote mean values $( \pm 1 \mathrm{SD}), N=3-$ 5 animals per group. 


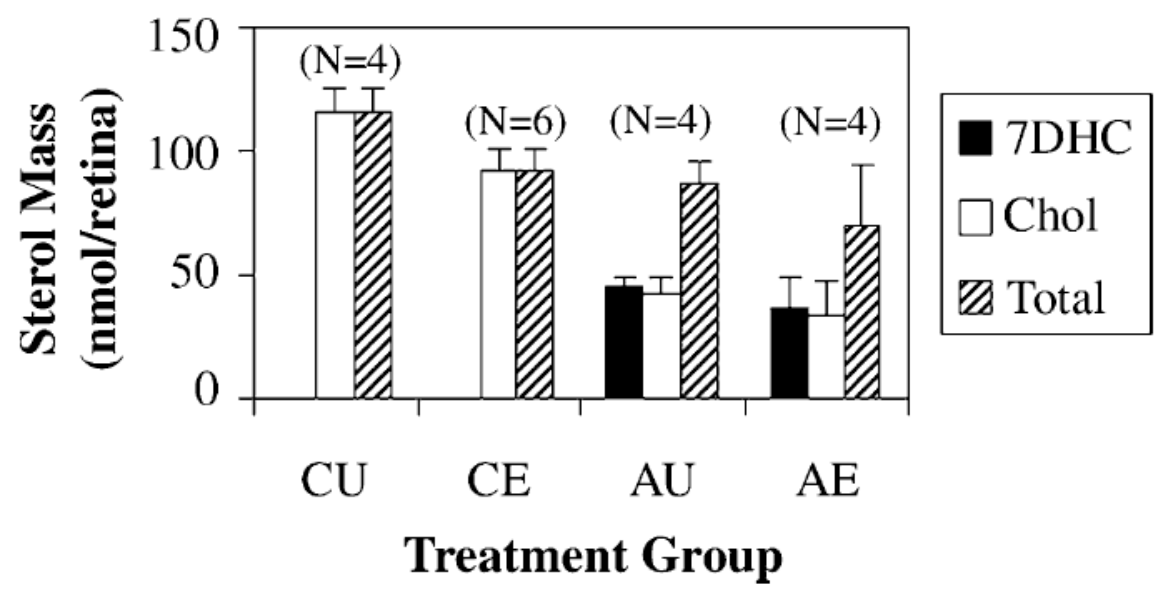

Fig. 2.

Sterol composition of retinas under various conditions, as measured by HPLC. CU, control, non-light-exposed; CE, control, exposed to intense green light; AU, AY9944-treated, nonlight-exposed; AE, AY9944-treated, exposed to intense green light. Note marked steady-state accumulation of 7DHC in AY9944-treated retinas, and its absence in control retinas (where cholesterol (Chol) represents $>99 \%$ of total sterols). 

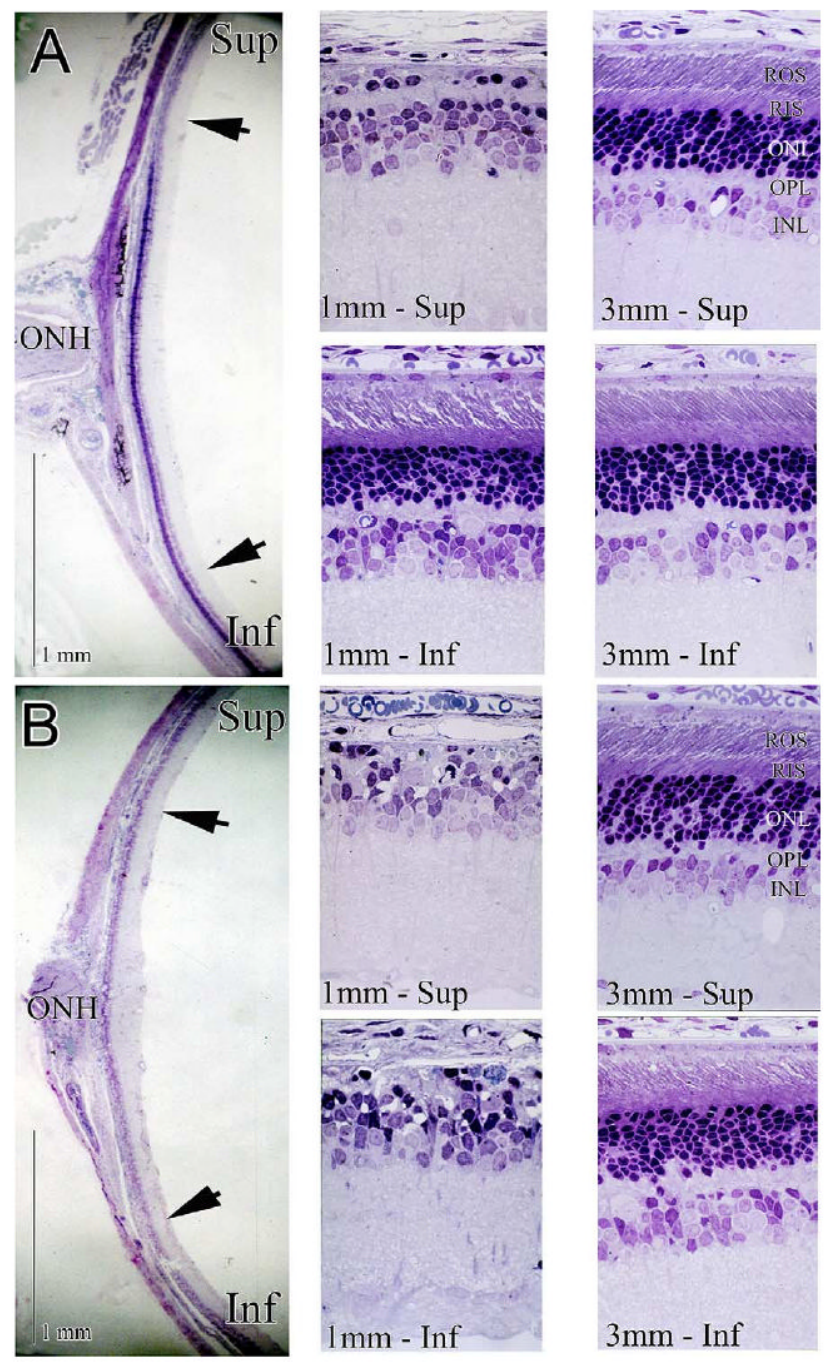

Fig. 3.

Histology of control and AY9944-treated retinas after exposure to intense green light. Images taken along the vertical meridian at low magnification (left-hand panels), as well as at higher magnification (4-panel cluster; images taken at 1 and $3 \mathrm{~mm}$, respectively, from optic nerve head $(\mathrm{ONH})$ in both the superior (Sup) and inferior (Inf) hemispheres). (A) Control rat retina exposed to intense green light (representative of CE group). Photoreceptor loss is restricted to the superior central zone; a few, mostly pyknotic rod nuclei remain apposed to the RPE in this zone, but the cells lack outer segments. (B) AY9944-treated rat retina exposed to intense green light (representative of AE group). Photoreceptor pyknosis and loss spans the entire central retina, both in the inferior and superior hemispheres. Arrowheads denote distance $1 \mathrm{~mm}$ from the ONH (per scale bar). ROS, rod outer segment layer; RIS, rod inner segment layer; ONL, outer nuclear layer; OPL, outer plexiform layer. 


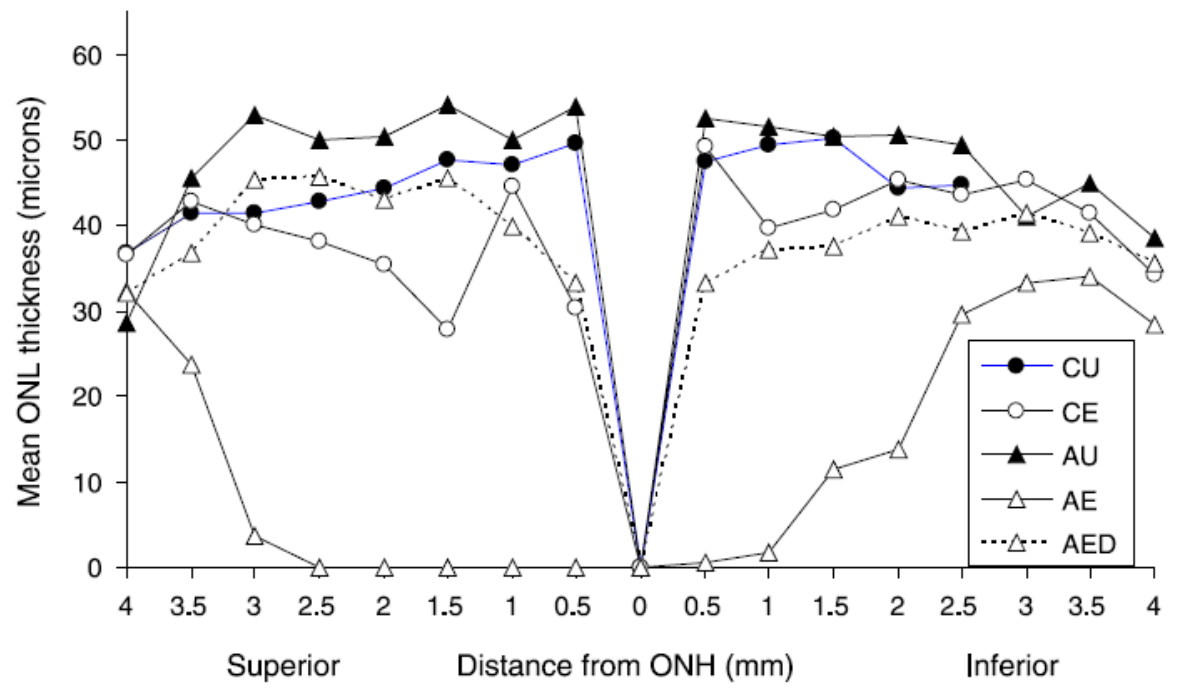

Fig. 4.

Photoreceptor survival, assessed by mean ONL thickness measurements, in rat retinas as a function of AY9944 treatment, intense light exposure, and antioxidant (DMTU) treatment prior to intense light exposure. C, controls; A, AY9944-treated; U, unexposed to intense light; E, exposed to intense light; D, treated with DMTU prior to light exposure. Note the significant potentiation of light damage by AY9944 administration (AE), compared to both non-lightexposed groups (AU, CU) and to the control light-exposed group (CE), and the substantial protection from light-induced damage afforded by DMTU pre-treatment (AED). 